tomine », ? OUG. hrpme "antomn, vb. II, to dote, be senile and feeble-minded; to harifa, a (haraf), vb. I, and
drivel, talk foolishly

haraf, $n$., feeble-mindedness, dotage, senility; childishness (of an old man)

harif and harfan, adj., feebleminded, doting; childish; $n$., dotard

harif, n., autumn, fall

harifi, adj, autumnal

harüf, pl. hirâf, ?abrifat, hirfan, $n$, young sheep, lamb, yearling; wether

huráfat, pl. -at, n., superstition; fable, fairy tale

stio. huräfi, adj., fabulous, fictitious, legendary

d'auc mahrafat, $n$., prattle, drivel, twaddle, bosh

péri tahrif. $n$. folly, delusion; foolish talk, drivel, twaddle, bosh, buncombe

mubarrif, adj., childish, foolish; ( $\mathrm{pl}$. -ün) prattler, chatterbox, windbag; charlatan

horf, hars horef "antomne», ETH. g. jib. hurof « débiter des absurdités, des

burt: l'annecen

«mouton de l'a

"brubis (?) »,

\section{Approaches}

\author{
to the
}

\section{Etymology of Arabic}

wipt- «sang coarpopita « escarre, SOUEN AIN 323,

edited by

STEPHAN GUTH

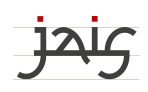

ISSN 0806-198X

Journal of Arabic and Islamic Studies • 17 (2017): 311-453 


\section{Contents}

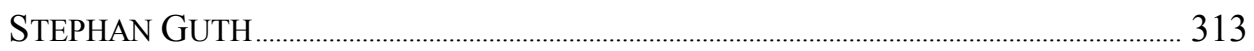

Introduction

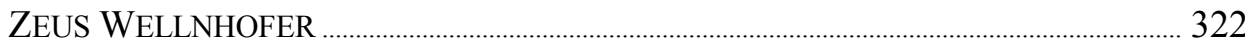

On Some Arabic Roots and Their Etymological Relevance

SIMONA OLIVIERI.

The ism in the Arabic Grammatical Tradition: Reflections on Its Origin and Meanings

STEPHAN GUTH

Biradicalist Mimophonic Triradicalism: Sounds, root nuclei and root complements in M. H. H. Gabal's 'etymological' dictionary

of Arabic (2012)

JEAN-CLAUDE ROLLAND

Éclats de roche : Une étude d'étymologie sur les noms de la pierre en latin, grec et arabe

LUTZ EDZARD

Notes on the Emergence of New Semitic Roots in the Light

of Compounding

FRANCESCO GRANDE

The Arabic Lexicographer Ibn Sīdah and the Notion of

Semantic Field

GiZEM IŞIK

Etymology and Polysemy: A Non-Objectivist Approach to the Domain of Vision in the Semitic Languages 


\title{
Éclats de roche : Une étude d'étymologie sur les noms de la pierre en latin, grec et arabe
}

\author{
JEAN-CLAUDE ROLLAND
}

Elles sont du début de la planète, parfois venues d'une autre étoile. Roger Caillois, Pierres

\begin{abstract}
Starting from the Latin cases of semantic parallelism, rūpēs \|rumpō and saxum \|secō, this study goes through a number of Arabic denominations of the stone to demonstrate that in Arabic too there is a strong relationship between those denominations and many roots that basically express an act of breaking or cutting something. The conclusions are the following:

- the Greek equivalents of "stone", the etymology of which is obscure, might have the same relationship with some Greek or Latin words expressing the act of breaking;

- Greek $\pi \dot{\varepsilon} \tau \rho \bar{\alpha}$ [pétrā] might be of Semitic origin.
\end{abstract}

Key words: Arabic, Greek, Latin, lexicology, etymology, stone, fragment, breaking, stroke, Bohas

Les associations sémantiques en rapport avec la pierre sont connues et banales : la première caractéristique de la pierre est sa dureté. Dure, elle est difficile à briser, à tailler, à sculpter. Ancêtre aussi bien du marteau que du couteau, elle va donc servir elle-même d'outil pour briser et tailler des objets moins durs qu'elle. Selon son volume elle est obstacle naturel sur la route ou matériau utilisé pour construire ces séparateurs que sont les parois, murs et murailles destinés à empêcher les uns d'entrer et les autres de sortir. Il ne faut donc pas s'étonner que le latin rūpēs rocher soit considéré par la tradition étymologique comme une forme sans infixe nasal issue de la même racine indo-européenne ${ }^{2}$ ayant abouti au verbe rumpō briser, rompre, sans qu'on sache si cette forme rūpēs est à l'origine active ou passive, autrement dit si le rocher latin est « brisant » ou « brisé ».

Plutôt « brisé » car la pierre, aussi arrondie soit-elle par l'érosion, est généralement perçue comme ce qu'elle est, c'est à dire un fragment, un éclat de roche. De taille réduite, elle sert de projectile, d'arme lancée par la main, la fronde ou la catapulte. Tout objet un peu dur lui est comparé ou assimilé : noyau, dent, fragment d'os, corail, coquille d'œuf, coquillage et même testicules. Sous forme de sable, de gravier ou de cailloux, elle est marque de stérilité, d'absence de végétation, de désert. Dite " précieuse » ou " semi-précieuse », elle orne le collier ou la bague. Elle est borne sur la route, statue monumentale, idole, menhir, dolmen, et tombe ou stèle au cimetière.

Cette étude se propose d'examiner brièvement dans une première partie ce qu'il en est—outre le cas de rūpēs, sur lequel nous ne reviendrons pas-des dénominations de la pierre en grec et en latin, pour la plupart d'origines obscures, et dans une deuxième partie,

1 ERNOUT \& MEILLET 1932/2000: 581.

2 *reup- ou *reub-, selon WATKINS 2000: 72. 
plus longue et plus élaborée, de faire le même inventaire pour l'arabe, en nous appuyant sur les travaux que Georges Bohas a consacrés au lexique du coup en général et de la coupure en particulier. Ces travaux nous autoriseront en effet à rattacher à ces notions primaires tout un réseau ${ }^{3}$ de notions dérivées qu'une approche naïve ne permettrait pas forcément de percevoir d'emblée et d'une façon aussi nette et rigoureuse. Aussi renverrons-nous à ce réseau chaque fois que la relation d'une notion dérivée avec les notions primaires risquerait de ne pas être évidente. On verra que notre étude des noms arabes de la pierre, initiée et éclairée par le parallélisme morphosémantique relevé dans la paire rūpēs // rumpō, pourrait bien en retour jeter quelque lumière sur certaines dénominations grecques ou latines aux origines jugées jusqu'à ce jour obscures.

\section{Les dénominations grecques et latines de la pierre}

1.1. latin saxum pierre, grosse pierre, roc, rocher

1.2. latin lapis pierre, pierre précieuse

1.3. grec $\lambda \hat{\theta} \theta$ os [líthos] pierre et пां́ $\rho \bar{\alpha}$ [pétrā] roche, rocher

\subsection{Latin saxum pierre, grosse pierre, roc, rocher}

De l'origine de ce mot—que la langue française conserve précieusement comme composant de quelques termes savants tels saxifrage et saxicole - voici ce que disent Ernout et Meillet dans leur Dictionnaire étymologique de la langue latine (DELL), p. 597 :

Pour la forme, saxum concorde avec le vieil islandais sax, le vieux haut allemand sahs " couteau, épée courte»; mais le mot germanique appartient à un groupe de noms indiquant des objets tranchants: vieux haut allemand sega, sego " scie », segesna, segansa « faux », etc. Les mots germaniques sont donc évidemment de la famille du latin secāre. Le latin saxum y peut aussi à la rigueur être rattaché, mais par un autre procès de sens : le rapport serait de même ordre que celui du latin rūpēs avec rumpō, du vieux slavon skala «pierre, rocher » avec le lituanien skeliù « je fends », etc.

Avec rūpēs // rumpō et saxum // secōo, nous voilà donc en présence, pour la seule langue latine, de deux cas d'un probable parallélisme sémantique pierre // porter un coup que la suite de cette étude aura pour objet de confirmer, d'expliquer, et d'étendre à d'autres langues que le latin. Sous le générique pierre, il faut comprendre que nous pourrons en rencontrer toutes les spécificités : rocher, caillou, gravier, sable, etc. ainsi que tout objet dur pouvant lui être comparé : noyau, dent, os, etc. Quant aux diverses et nombreuses spécificités représentées par le générique porter un coup, on les trouvera énumérées, ordonnées et explicitées dans le réseau ad hoc situé en annexe. Citons-en néanmoins ici quelques-unes : frapper, battre, couper, fendre, percer, briser, tuer, repousser, chasser, etc.

3 Voir Annexe.

jais • 17 (2017): 377-406 


\subsection{Latin lapis pierre, pierre précieuse}

D’après le DELL (p. 340), le mot lapis, -idis - d'où viennent les mots français lapider, lapidaire, etc.- désigne une pierre, ou tout objet en pierre ou qui rappelle la pierre : borne miliaire ou frontière, monument funèbre, statue, et également pierre précieuse. En bref, par opposition à saxum qui n'est que la pierre brute, lapis, c'est la pierre travaillée. En ce qui concerne l'étymologie du mot, Ernout et Meillet ne s'aventurent pas à proposer la moindre hypothèse. Ils rejettent même un rapprochement avec le grec $\lambda$ É $\pi \alpha \varsigma$ lépas rocher $n u$ car ce mot, disent-ils, est « loin pour le sens » et « suspect d'appartenir à la famille de $\lambda \dot{\varepsilon} \pi \omega$ lé-

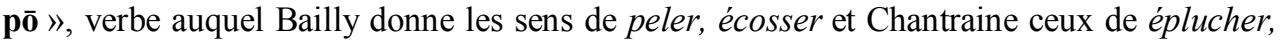
enlever l'écorce, donner une râclée, manger. Le même Chantraine aligne sa position sur

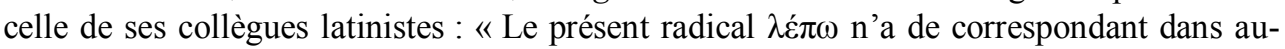
cune autre langue. [...] Terme de substrat pour Beekes, Orbis 1971, 132. » Dans la notice consacrée à $\lambda \hat{\varepsilon} \pi \alpha$, Chantraine évoque cependant la possibilité de rapprocher ce mot soit de lapis, soit de $\lambda \dot{\varepsilon} \pi \omega$, sans se prononcer clairement pour l'une des deux hypothèses, ni d'ailleurs les rejeter.

Moins savant et plus imprudent sans doute que ces éminentes autorités, nous nous permettons néanmoins de contester leurs deux arguments :

- le premier car entre une pierre, aussi travaillée soit-elle, et un rocher, il n'y a tout de même pas un énorme fossé sémantique. C'est aussi l'avis de Michiel De Vaan ${ }^{4}$ dont voici une copie de la notice qu'il consacre à lapis : ${ }^{5}$

lapis, -dis 'stone, pebble' [m. d] (Naev.+)

Derivatives: lapideus 'of stone, stony' (P1.+), lapidārius 'of stone-cutting' (P1.+), lapidōsus 'stony' (Varro+), lapillus 'small stone' (Varro+); lapicīda 'stonecutter' (Varro+), lapicīdīnae [f.pl.] (Cato+), lapidicinnae [f.pl.] (Varro+) 'stonequarries'.

PIt. *la/eped-. It. cognates: U. vapeře [loc.sg.], vapefem, uapefe [acc.pl. + -en], uapersus [abl.pl.], uapersusto [abl.pl. + -to] 'stone seat'. Uncertain: SPic. vipetí, repetín, vepeten, iepeten, yepetin [loc.sg. + -en]* 'monument?' <*-eto-.

IE cognates: Gr. $\lambda \varepsilon ́ \pi \alpha \varsigma$ [n.] 'bare rock, mountain', $\lambda \varepsilon \pi \alpha ́ \varsigma,-\alpha ́ \delta o \varsigma$ [f.] 'limpet', $\lambda \varepsilon \pi \alpha ́ \delta \varepsilon \varsigma$ 'molluses which stick to rocks' (Hsch.).

Probably a Mediterranean loanword of the structure *lVpVd-.

Bibl.: WH I: 761, EM 340f., IEW 678, Schrijver 1991: 486, Untermann 2000: 823f., 838 .

Notons en effet, ce deuxième cognat d'une accentuation et d'un genre grammatical diffé-

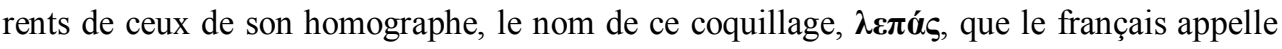
tout simplement lépas ou lepas: c'est un coquillage univalve qui s'attache aux roches et aux bateaux et dont la dure carapace présente, comme d'ailleurs celle de tous les coquillages, une évidente ressemblance avec la pierre ;

4 DE VAAN 2008.

5 Le contenu de cette notice est partiellement repris dans celle que Douglas HARPER consacre à lapideous dans son propre site Etymology on Line (ETYMONLINE). 
- et nous contestons aussi le deuxième argument car $\lambda \dot{\varepsilon} \pi \omega$ a une très bonne raison d'être apparenté à $\lambda \dot{\varepsilon} \pi \alpha \varsigma$, à savoir le fait qu'il entretient avec ce mot la même relation morphosémantique que nous avons déjà rencontrée deux fois dans les paires rūpēs // rumpō et saxum // secō : les divers sens de ce verbe tels que donnés par Bailly et Chantraine se trouvent en effet être des déclinaisons de la notion porter un coup (voir annexe, A.1.3.3).

Une fois admise la parenté de lapis avec $\lambda \dot{\varepsilon} \pi \omega$ par l'intermédiaire de $\lambda \hat{\varepsilon} \pi \alpha \varsigma$, on peut aller plus loin. Il suffit de consulter la longue notice que Chantraine consacre à ce verbe et à ses dérivés où il n'est question que d'écailles, d'écales, de coquilles, de cosses, d'éclats de métal, de pelures d'ognon, etc. Sans surprise, on y trouvera un autre cognat : $\lambda \dot{\varepsilon} \pi \rho \alpha$ lèpre, maladie «caractérisée à un premier stade par la formation d'écailles, de tubercules et de pustules à la surface de la peau ». (TLF).

À ce stade où il semble acquis que les radicaux *lap- et *lep- n'en forment finalement qu'un seul—qu'à l'instar de de Vaan on pourrait d'ailleurs écrire *la/ep- -, il est tentant de nous tourner à nouveau vers le latin où nous attend lepus, -oris lièvre, et vers le français où nous attend lapin, deux mots que l'étymologie traditionnelle traite curieusement par des voies différentes quand ce n'est pas par un expéditif « Emprunt à une langue méditerranéenne ». Quel rapport ces animaux entretiennent-ils donc avec la pierre ? Aucun, a priori, même si, via le français lapereau qui se dit laparo en portugais, on a tenté de faire remonter lapin au thème ibéro-roman *lappa- «pierre plate» qui serait à l'origine du portugais lapa « roche saillante ; caverne, grotte », attesté en 907 dans un texte latin, ${ }^{6}$, au prétexte que les lapins établissent leur repaire souvent dans la terre couverte de pierres.

Sceptique quant à cette hypothèse — on le serait à moins ! -, Pierre Guiraud ${ }^{7}$ propose quant à lui un croisement du latin lepus avec le verbe français lapper « manger avec avidité, être gourmand». Nous lui donnons raison mais en partie seulement: en observant la forme et le fonctionnement des incisives caractéristiques de ces deux espèces, nos ancêtres avaient qualifié plus justement que par notre fautif « rongeurs $»^{8}$ ces animaux qui «mordaient à belles dents » leur nourriture : le lièvre, baptisé lepus en Sicile, d'après Varron, tient très probablement son nom du verbe grec $\lambda \dot{\varepsilon} \pi \omega$; et le lapin son nom français tout aussi probablement d'une variante latine *lapo disparue mais dont la réalité est attestée par l'existence du substantif lapis, lequel devait être à *lapo ce que $\lambda \dot{\varepsilon} \pi \alpha \varsigma$ est à $\lambda \dot{\varepsilon} \pi \omega$.

Il est bien possible que ces radicaux ne soient que méditerranéens car on ne leur voit pas de cognats dans d'autres langues que le grec et le latin, mais il est plus que probable que tous les mots que nous venons de voir appartiennent à une petite famille dont l'invariant sémantique de base est porter un coup, c'est à dire le même qui a été relevé par Ernout et Meillet dans les paires rūpēs // rumpō et saxum // secō. Autour de la volcanique Méditerranée, on n'utilisait pas le même vocabulaire mais on avait la même vision de la pierre : un fragment de roche, une écaille de la croûte terrestre.

6 Le Trésor de la langue française (TLF).

7 Dictionnaire des étymologies obscures.

8 Les Léporidés sont maintenant classés dans l'ordre des Lagomorphes. 


\subsection{Ní⿴os [líthos] 'pierre' et métpā [pétrā] 'roche, rocher'}

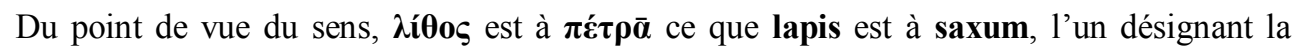
pierre travaillée et l'autre la pierre brute.

Nous devons au premier l'un des composants de nos lithographie, mégalithe et autres termes savants, et au deuxième - via le latin petra très tôt emprunté au grec_-la famille de pierre avec ses deux branches de dérivés populaires (ex. pierreux) et savants (ex. pétrifier, pétrole).

Nous n'en savons guère plus sur ces deux vocables. Leur origine est inconnue. Chantraine ne s'y attarde pas; sa notice Étymologie du premier se résume à un mot : « Ignorée ». Pour le deuxième, ce sera une phrase : «Parmi les étymologies énumérées chez Frisk, aucune n'est satisfaisante. » Notre méconnaissance de la langue allemande nous interdit de consulter l'ouvrage de cet auteur et c'est bien dommage car nous aurions souhaité vérifier si les hypothèses que nous nous apprêtons à proposer sont vraiment nouvelles.

Nous aurons effectivement, en conclusion de notre étude, des propositions étymo-

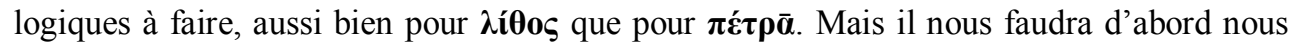
pencher assez longuement sur les dénominations arabes de la pierre. Voyons si le détour en vaut la peine.

\section{Les dénominations arabes de la pierre}

2.1. hağar pierre ${ }^{9}$

2.2. ḥașan (ḥașà) pierraille, cailloux

2.3. qaự sable et petits cailloux, gravier

2.4. raml sable

2.5. șahr roc, rocher

2.6. ğundul ${ }^{10}$ ou ğandal pierre, rocher

2.7. Synthèse

\section{Remarques générales préliminaires}

Tels la matière qu'ils dénotent, les six mots vedettes que nous avons choisis ont perduré à travers les siècles. Ils restent aussi usuels en arabe moderne qu'ils le furent en arabe classique, sans changement de forme ni de sens. C'est la raison pour laquelle nous avons choisi de les privilégier. Ce n'est pas pour autant que, dans les lignes qui suivent, nous passerons sous silence les mots de l'arabe classique devenus obsolètes, car ils ont une fonction essentielle, celle de révéler des relations sémantiques que le seul examen du lexique moderne aurait laissées dans l'ombre.

Notre étude porte exclusivement sur les dénominations de la pierre, non sur les racines exprimant la notion de porter un coup en ses diverses déclinaisons, travail dont la plus grosse partie a été faite par Georges Bohas ou par des étudiants sous sa direction. Nous nous sommes efforcé de trouver à quelle famille morpho-sémantique appartenait chacun de

\footnotetext{
9 On retrouve ce mot dans le toponyme espagnol Guadalajara, littéralement «l'oued des pierres ».

10 À ne pas confondre avec l'emprunt ğundūl « gondole ».
} 
nos mots titres. Ce faisant, nous avons le plus souvent constaté la présence du parallélisme pierre // porter un coup au sein des racines inventoriées, mais nous n'avons pas rejeté celles qui ne présentaient qu'un seul des composants dans la mesure où elles étaient dotées des caractéristiques morphologiques de la famille considérée, renforçant par leur seule existence celle de la famille toute entière.

Dans la théorie de Bohas, une famille morphosémantique arabe se caractérise par la présence, au sein des racines qui la composent, d'un "étymon" bilitère de type $\left\{\mathbf{C}^{1}, \mathbf{C}^{2}\right\}$ porteur d'une même charge sémantique. La troisième consonne d'une racine trilitère a le statut de crément - préfixe, suffixe ou infixe — et la place des consonnes n'est ni fixe ni ordonnée. Les racines quadrilitères ont deux créments ou sont le résultat du croisement de deux étymons synonymes ou complémentaires, soit $\left\{\mathbf{C}^{1}, \mathbf{C}^{2}\right\}+\left\{\mathbf{C}^{3}, \mathbf{C}^{4}\right\}$. Les racines trilitères elles-même peuvent être le résultat d'un croisement d'étymons, soit $\left\{\mathbf{C}^{1}, \mathbf{C}^{2}\right\}+$ $\left\{\mathbf{C}^{2}, \mathbf{C}^{3}\right\}$. On verra que nous avons très souvent pu constituer dans chaque famille de nombreux sous-groupes de racines composées des mêmes consonnes permutées, au point que ces racines apparaissent parfois comme de simples variantes les unes des autres.

$>$ Les glides $\mathbf{w}$ et $\mathbf{y}$ n'étant généralement pas considérés comme des radicales, les racines trilitères qui en comportent révèlent d'emblée leur étymon. C'est aussi le cas des racines dites "sourdes" de type $C^{1}-C^{2}-C^{2}$ et des racines quadrilitères à couple redoublé de type $C^{1}$ $\mathrm{C}^{2}-\mathrm{C}^{1}-\mathrm{C}^{2}$. Ces racines sont dites "non ambigües". Toutes les autres sont dites "ambigües" car elles pourraient être construites sur n'importe lequel des trois étymons théoriquement possibles : $\left\{\mathbf{C}^{1}, \mathbf{C}^{2}\right\},\left\{\mathbf{C}^{1}, \mathbf{C}^{3}\right\}$ ou $\left\{\mathbf{C}^{2}, \mathbf{C}^{3}\right\}$. Seul le sémantisme de la racine en question permet alors de décider lequel des trois étymons est à privilégier, et par là à quelle famille morphosémantique peut être associée cette racine ou au moins certains de ses dérivés, le lexique arabe n'étant pas exempt de cas d'homonymie.

La théorie de Bohas permet d'opérer des regroupements lexicaux à un niveau submorphémique à partir des traits phonétiques qui caractérisent les diverses consonnes de l'arabe et de constituer ainsi des "matrices" phoniques d'étymons synonymes. Chaque matrice a donc son propre invariant notionnel et sa propre arborescence de ramifications sémantiques. Au moins trois des matrices révélées à ce jour - dont la matrice $\mathrm{n}^{\circ} 5$-ont porter un coup ou des coups comme invariant notionnel, ce qui a permis à Bohas d'élaborer le document que nous avons mis en annexe pour nous y référer en cas de besoin. Cela étant, nous avons pris le parti d'en rester généralement au niveau morphémique, laissant à d'autres, plus savants en phonétique et plus hardis, le soin de constituer sur ces bases des familles plus vastes mais en moins grand nombre. On pouvait néanmoins supposer que certains de nos mots titres relèveraient d'une même matrice : on vérifiera que trois d'entre eux relèvent effectivement de la matrice $\mathrm{n}^{\circ} 5$ et un quatrième de la matrice $\mathrm{n}^{\circ}$ 1 , deux des trois matrices ayant porter un coup ou des coups comme invariant notionnel.

\section{1. hağar pierre}

À tout seigneur, tout honneur. Nous nous devions de commencer cette étude par le substantif qui reste le terme le plus usuel pour désigner la pierre. 


\subsubsection{Morphosémantisme de la racine}

Sous l'entrée $\sqrt{ }$ g̣ğr du dictionnaire de Kazimirski, on trouvera, entre autres, les items suivants :

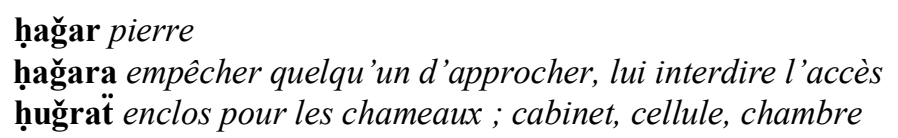

On constate d'emblée dans cette racine la présence du parallélisme sémantique pierre // porter un coup. Les sens du verbe et ceux de ḥuğrä̈ relèvent en effet des items A.7.1. pousser, repousser et A.7.2. protéger conserver, garder du réseau sémantique de porter un coup (voir annexe).

On pourrait en rester là et passer au mot titre suivant. Mais les plus sceptiques de nos lecteurs risqueraient de critiquer la légèreté avec laquelle nous traiterions la première de nos vedettes en nous appuyant sur le seul fait que les notions de pousser et de protéger auraient quelque chose à voir avec porter un coup ou couper. Et ils auraient raison. Voyons donc d'abord ce qu'il en est de la morphologie et des liens de parenté que la racine $\sqrt[h]{\mathbf{g} g \mathbf{r}}$ entretient avec d'autres racines arabes ou plus généralement sémitiques.

La première racine qu'il vient à l'esprit de rapprocher est V̆̆g̣ blesser (à l'arme blanche), faire une coupure dans la chair : d'aucuns diraient que les deux racines pourraient effectivement être apparentées, au moins par le forme, au prix d'une simple métathèse. Nous préférons dire que ces deux racines ont peut-être le même étymon $\{\check{\mathbf{g}}, \mathbf{r}\}$ affecté du même crément hạ, en position initiale ou préfixée dans l'un, en position finale ou suffixée dans l'autre. Il se trouve que, dans la théorie de Bohas, cet étymon, doté de la charge sémantique porter un coup, relève de la matrice 5 \{[coronal],[dorsal]\} porter un coup ou des coups. Voyons donc ce qu'il en est de la réalisation de cet étymon dans une racine non

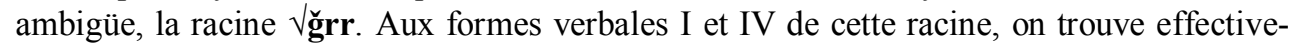
ment les significations suivantes :

ğarra - I. extraire, arracher, faire sortir ; fendre la langue à un petit chameau - IV. porter un coup de lance

Or, sous cette même entrée, dans le dictionnaire de Kazimirski, on trouve également :

ğarr coquillages ou autres petits objets qu'on attache au cou des animaux en guise d'ornement - ğarrārä̈t terrain déprimé, encaissé, couvert de cailloux ${ }^{11}$

ğarağ sol dur et rocailleux n'est à l'évidence qu'une extension du radical ğr-par redoublement de la première radicale.

On peut ajouter le tigré gərgər rocher, falaise, trouvé dans le DRS, fasc. 3, p. 181, et l'amharique gurangur terrain pas très pierreux... mais quand même un peu! (id. p. 189).

Ce sont des racines quadrilitères obtenue par redoublement du radical GR-, plus un infixe nasal pour la deuxième.

11 Curieusement, ne sont signalés dans le DRS sous la racine GRR (fasc. 3: 191-192), ni les sens du verbe fendre la langue à un petit chameau - IV. porter un coup de lance, ni les deux substantifs que nous donnons ici, alors que ces données sont pour nous, on le voit, essentielles. 
Voilà donc qu'à l'image de ce que nous avons constaté en grec et en latin, nous trouvons associés sous une même racine sémitique, extensions comprises,

- un verbe exprimant la coupure,

- un nom désignant un rocher ou une falaise,

- plusieurs noms désignant un sol dur, un terrain pierreux, rocailleux ou caillouteux,

- et un nom désignant des coquillages.

S'il ne s'agit que d'une coïncidence, avouons qu'elle est pour le moins troublante.

À la lumière de ce qui précède, nous ne pensons pas qu'il soit nécessaire de chercher dans ḥağar un autre éymon que $\{\check{\mathbf{g}}, \mathbf{r}\}$. Ne disposant pas d'autres racines non ambigües construites sur cet étymon à examiner, voyons donc ce qu'il en est des racines ambigües.

\subsubsection{Racines ambigües probablement apparentées}

Notre hypothèse sera confortée par la présence du parallélisme pierre // couper dans de nombreuses racines ambigües. ${ }^{12}$ Certaines sont isolées ${ }^{13}$ mais la plupart constituent des sous-groupes autour de leurs consonnes communes permutées; c'est par elles que nous commencerons, le crément en italiques permettant de les différencier :

\section{- Groupe ğ-r-b}

ğurūb blocs de pierre servant d'assise (DRS, fasc. 3, p. 178) - ğarbā' (terre) frappée de sécheresse et de stérilité

sudarabique ğrb pierre brute (DRS, id.)

ğarab gale $^{14}$

hirğāb grand de taille (homme) - Pour la forme, cette racine quadrilitère peut être

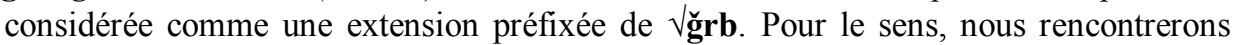
plus loin d'autre cas où la pierre est non seulement synonyme de dureté mais aussi de solidité et par extension de force, de grandeur, de grosseur, d'épaisseur.

syriaque garb dūdā morceau de rocher (DRS, id., p. 179)

amharique g"arbatța inégal (terrain) ; noueux (bois) (DRS, id.)

\section{- Groupe ğ-r-d}

ğurdä̈ sol uni et nu, où rien ne croît - Implicitement, c'est un terrain qui s'apparente à la pierre.

ğarada oter, enlever (p. ex. les feuilles des arbres, la peau, etc.)

tigré gārdādā ronger

Voir annexe A.1.3.3. Pour la peau qui pèle, cf. grec $\lambda \dot{\varepsilon} \pi \omega$ et $\lambda \dot{\varepsilon} \pi \rho \alpha$.

12 Le Dictionnaire des racines sémitiques, fasc. 3: 175 et suivantes, signale bien que de nombreuses racines en GR- ont la valeur couper > enlever, arracher, casser, etc. mais il ne fait pas de lien avec plusieurs désignations de la pierre qu'on trouve sous ces mêmes racines.

13 Voir plus bas, note 16.

14 Cette maladie de la peau nous rappelle que nous avons plus haut rencontré $\lambda \varepsilon ́ \pi \rho \alpha$ lépra dont l'équivalent arabe est baraṣ. On notera la présence de l'étymon $\{\mathbf{b}, \mathbf{r}\}$ dans ces deux termes. Autrement dit, ğarab résulte probablement du croisement des étymons $\{\breve{\mathbf{g}}, \mathbf{r}\}$ et $\{\mathbf{b}, \mathbf{r}\}$. 
Notons également que l'arabe gurad désigne les muridés...

ğadara faire élever une muraille autour de qqch - ğadr mur, muraille - ğadīrä̈ enclos fait de pierres pour les bestiaux

Mur et muraille séparateurs, et enclos explicitement "fait de pierres".

ğadara avoir des pustules, s'en couvrir; contracter des callosités

Comme nous l'avons vu en 1.2., nous retrouvons ici les pustules de la lèpre, de la peau malade, qui pèle. (Cf. ğarada ci-dessus). On sait par ailleurs que cal et caillou ont une même origine celtique.

\section{- Groupe ğ-r-z}

ğariza être épais et dur

ğaraza couper, retrancher

hébreu garzen hache, pic à roc (DRS, id., p. 185)

ğazīrä́ $̂ l e$

Une île est bien un fragment séparé du continent, avec un sommet rocheux ayant résisté à l'érosion.

ğazara égorger, tuer (une pièce de bétail)

\section{- Groupe ğ-r-š}

hadrawi ğarī̌sa meule à bras

L'hadrawi nous procure le nom manquant, celui de cet antique outil en pierre.

ğaraša casser, piler gros, grossièrement - ğaraša VIII. enlever, emporter, happer

Tous ces verbes français sont des déclinaisons de porter un coup. Nous n'aurons désormais plus besoin de le rappeler.

hašrağ source dans un terrain couvert de cailloux; cailloux

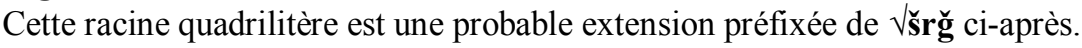

šarağa - VII. se fendre ; être gercé, crevassé

Sans commentaire.

\section{- Groupe ğ-r-f}

ğurf, ğuruf terre que l'eau du torrent ronge et emporte; berge, bord rongé par l'eau; pl. ğurüf pierre, digue en pierre

Notons cette première apparition de berge, bord. Il y en aura d'autres.

ğarafa enlever, emporter tout, en balayant, d'un coup de balai ou de pelle

Voir plus haut ğaraša. Sans autre commentaire.

fağara faire jaillir l'eau en fendant un rocher

farağa fendre, pourfendre

Notre parallélisme n'est avéré dans aucune de ces deux racines quoique la biblique présence du rocher dans la signification du premier verbe ne soit certainement pas fortuite. Quant au deuxième verbe, il semble bien n'être qu'une variante du premier. Quoi qu'il en soit, nous nous devions de signaler l'existence de ces deux racines au sein du groupe ğ-r-f. 


\section{- Groupe ğ-r-m}

ğaram noyau de datte - hébreu gerem, syriaque $\mathbf{g}^{\mathrm{e}} \mathbf{r u ̄ m a ̄}$ os, noyau

ğarama couper, retrancher; prendre, enlever

Sans commentaire.

ğammara jeter des cailloux - ğimār petits cailloux qu'on jette, pendant les fêtes de la Mecque, à la vallée de Muna, selon les cérémonies d'usage, comme pour lapider Satan; pierre sépulcrale - ğumrä̈ braise allumée, charbon; espèce de pustules qui se déclarent sur le corps ; cailloux, petits cailloux dont le lit des torrents est jonché

ğammara couper un palmier

Sans commentaire.

ğumhūr monticule de sable

ğamhara enlever la meilleure partie d'une chose

Cette racine quadrilitère est une probable extension de $\sqrt{\text { ğmr }}$ par l'infixe guttural $\mathbf{h}$. On peut aussi la considérer comme relevant du groupe $\breve{g}-\mathbf{r}-\boldsymbol{h}$ avec infixe nasal $\mathbf{m}$. Nous la retrouverons donc plus loin.

rağama lapider - rağam, ruğum, ruğmä̈ tas de pierres jetées sur un cadavre - riğām grande pierre ; pierre attachée au bout d'une corde pour agiter la vase au fond du puits rağama éloigner, repousser qqn à coups de pierres ; couper, arracher, séparer du tout Sans commentaire.

marğān corail

mariğa être en désarroi, se déranger, se désorganiser - IV. violer, enfreindre un pacte, une alliance

Le nom marğān a fait l'objet d'un article de Michel Masson ${ }^{15}$ dans lequel l'auteur démontre l'origine sémitique de ce mot et son rattachement à la racine $\sqrt{ } \mathbf{m r g}$ mais par un autre cheminement que le nôtre. Les deux démonstrations se confortent plus qu'elles ne s'opposent.

La locution nominale harğ wa-marğ grande confusion, justement citée par Masson, nous fournit la meilleure transition possible vers le groupe suivant.

\section{- Groupe ğ-r-h}

ğumhūr monticule de sable

ğamhara enlever la meilleure partie d'une chose

Racine quadrilitère vue plus haut dans le groupe ğ-r-m.

Nous n'avons dans ce groupe pas d'autres racines présentant le parallélisme pierre // couper mais nous en avons plusieurs présentant l'un ou l'autre de ses composants :

Deux racines trilitères :

harğ désordre, troubles et meurtres

Signalé plus haut en compagnie de marğ dans la locution harğ wa-marğ. Pour le lien sémantique avec porter un coup, voir annexe, B.2.

15 MASSON 2013b.

jais • 17 (2017): 377-406 
hağara rompre avec qqn et s'éloigner; abandonner, délaisser (une chose) - hiğrä rupture, séparation, cessation de rapports entre les personnes qui ont été amies; éloignement, départ; manière de se séparer, de rompre ses relations avec qqn; émigration d'un pays dans un autre; Hégire.

Quatre racines quadrilitères :

hağāris verglas

Nous rencontrerons d'autre cas où gel, glace et verglas sont assimilés à la pierre.

hirğās gros, corpulent

Même composition consonantique que la précédente.

harāğīil grands, longs (hommes) ; gros, énormes (chameaux)

ğawhar bijou, pierre précieuse

À notre avis, il existe probablement deux ğawhar homonymes, celui-là, d'origine sémitique et apparenté à ḥağar, et ğawhar essence, substance, nature, qui est un emprunt au pehlevi gōhr, id., d'où le persan gawhar.

- Racines isolées ${ }^{16}$

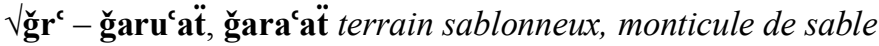

hébreu gāra' tailler la barbe, raccourcir, ôter - syriaque $\mathbf{g}^{\mathrm{e}} \mathbf{r a}^{\mathrm{c}}$ tondre

$\sqrt{ } \mathbf{r t g}$ - ritāğä̈ $r o c$, rocher

ratağa fermer, barricader une porte

sudarabique gerḥét dune de sable

ğaraḥa blesser (à l'arme blanche), faire une coupure dans la chair

Le parallélisme est si évident dans ces paires qu'elles se passent de commentaire.

\subsubsection{Racines ambigües avec le seul sémantisme pierre}

Pour les deux racines ci-dessous - qui n'en font peut-être qu'une - nous avons bien le premier composant du parallélisme, la pierre, mais, sauf erreur, nous n'avons pas le second :

ğarala être pierreux et dur - ğaral terrain pierreux où il se trouve des arbres - ğaril pierreux et dur (terrain)

ğarwal, ğurawil, ğurūlä̆ terrain pierreux ; pierres, rochers

Cette racine quadrilitère peut être considérée comme une extension infixée de $\sqrt{\text { ğrl. }}$

$\checkmark \mathbf{r g ̆ \mathbf { l }}$ - 'arğal dur et raboteux, semé de pierres (sol)

harāğĭl grands, longs (hommes); gros, énormes (chameaux)

Cette racine quadrilitère, déjà vue plus haut dans le groupe ğ-r- $\boldsymbol{h}$, peut aussi être considérée comme une extension préfixée de $\sqrt{ }$ rğl.

On voit que 'arğal a de fortes chances d'être apparenté à ğaril plutôt qu'à la racine $\sqrt{ }$ rğl homonyme dont le sémantisme est pied. Ce n'est qu'artificiellement et par étymologie populaire qu'on le rattache à cette dernière en disant, comme on le lit, non sans sourire,

16 «Racines isolées » signifie que je n'ai pas trouvé d'autres racines avec le même crément pour pouvoir constituer un groupe. 
chez Kazimirski, “... et qui endommage promptement le pied du piéton.” Cela dit, c'est tout de même une allusion à l'aspect tranchant de certaines pierres aux arêtes particulièrement aiguisées.

\subsubsection{Récapitulons}

Page|388 On trouve en arabe la séquence bilitère -ğr- (plus rarement -ř̆-) dans un nombre relativement important de racines dont un verbe dérivé a fondamentalement un ou plusieurs des sens suivants : couper, fendre, briser, peler, etc., ou un sens dérivé : arracher, oter, enlever, éloigner, repousser, blesser, tuer, etc. L'une de ces racines est $\sqrt{ } \mathbf{h} g ̆ \mathbf{r}$ dont le verbe ḥağara a le sens de empêcher quelqu'un d'approcher.

Dans un nombre inférieur-mais qui reste significatif-de ces mêmes racines, il se trouve un ou plusieurs substantifs qui désignent la pierre ou un objet assimilable à la pierre. L'une de ces racines est $\sqrt{ }$ ḥğr sous laquelle on trouve le substantif hạağar, le terme le plus usuel pour désigner ce minéral.

On peut raisonnablement penser qu'il en va de la paire ḥağar // ḥağara comme de rūpēs // rumpō et saxum // secō : c'est un même rapport sémantique pierre // couper qui relie, dans chacune de ces paires, un terme à l'autre. La perception de la pierre est la même en latin et en arabe : c'est un fragment détaché de la roche.

Voyons ce qu'il en est de nos autres mots vedettes.

\section{2. ḥașan/ḥașà pierraille, cailloux}

\subsubsection{Morpho-sémantisme de la racine}

ḥașan/ḥașà pierraille, cailloux; calcul, gravelle, pierre dans les reins ou dans la vessie hasạà jeter des cailloux contre qqn

Cette racine, qui se trouve être non ambigüe, relève de l'étymon $\{\mathbf{h}, \mathbf{s}\} .{ }^{17}$ Et notre parallélisme sémantique pierre // porter un coup y est patent.

Avec plusieurs autres racines non ambigües, et dont certaines présentent notre parallélisme, elle est en bonne compagnie :

ḥuṣāṣā’ terre, sol // ḥașṣa raser le poil, les cheveux

ḥāṣa $\boldsymbol{i}$ s'écarter, s'éloigner de la ligne droite ; éviter, fuir qqn

ḥașā u retenir, empêcher qqn d'approcher de qqch

hișhị terre ; pierres

wạ̣s petits boutons qui viennent sur visage d'une jeune et jolie fille

Une affection de la peau...

șāḥä sol nu, stérile - șawḥ berge, bord d'une rivière, bord élevé comme un mur // ṣāḥa fendre

Notons ce șawh berge, bord d'une rivière, nous en reparlerons.

17 Dans la théorie de Bohas, cet étymon est traité mais avec d'autres charges sémantiques. Voir notamment BOHAS \& BACHMAR 2013: 71 et BOHAS \& SAGUER 2014: 279. 


\subsubsection{Racines ambigües probablement apparentées}

On verra que la plupart des items ci-après se passent de commentaires.

\section{- Groupe ḥ-ș-b}

ḥaṣab cailloux - ḥaṣabä̈ caillou ; rougeole

Une autre affection de la peau...

hașaba joncher, couvrir de petits cailloux; jeter, lancer des petits cailloux sur qqn; frapper qqn ; abandonner qqn, se séparer de son compagnon

șaḥaba écorcher, dépouiller de sa peau

\section{- Groupe ḥ-ș-r}

ḥuṣr constipation

Dureté du ventre.

ḥișār enclos

Cf. ḥuğrä̈ enclos pour les chameaux

șaḥrā' champ dépourvu de végétation; au pl. Sahara

șụrä fente, crevasse dans un rocher

șarhäat sol dur

ḥarașa fendre, percer (la peau), casser (la tête)

\section{- Groupe ḥ-ș-f}

hafṣ noyau

ḥafașa jeter, lancer

ṣuffāh tablette mince en pierre (ardoise) - șafīh rocher en mer

șafīh sabre à large lame

ḥașafa éloigner, mettre à une grande distance ; ḥașifa être galeux

Une autre affection de la peau...

\section{- Groupe h-ș-m}

hașīm petits cailloux

ḥaṣama casser, briser

șimḥầ' sol dur et raboteux - șamūḥ dur (sabot) - 'aṣmạ̣ fort, brave, hardi

\section{- Racine isolée}

ḥașana être fort - ḥașīn fort, fortifié ; solide (cuirasse)

'ạ̣șinä̈ fers, pointes des lances 


\section{3. qaḍd petits cailloux, gravier}

\subsubsection{Morpho-sémantisme de la racine}

qaụḍ sable et petits cailloux, gravier - qiḍ̣ää grosse pierre informe ; sol jonché de petits caillous

qaḍ̣a percer, perforer une perle ; amincir un pieu, le tailler à l'extrémité

Cette racine, qui se trouve être non ambigüe, relève de l'étymon $\{\mathbf{q}, \mathbf{d}\},{ }^{18}$ ce qui est confirmé par sa présence au sein de la matrice 5 \{[coronal],[dorsal]\} porter un ou des coups de Bohas.

Notre parallélisme sémantique y est patent.

Avec plusieurs autres racines non ambigües qui présentent toutes notre parallélisme, elle est en très bonne compagnie :

qaḍqāọ sol égal et uni

qaḍqạ̣a fracasser, broyer (en parlant du lion et des os de sa proie)

qaḍ̣̣̂̄' solide (cuirasse)

qaḍà $\boldsymbol{i}$ décider, trancher

qị̄ää fragment, éclat d'os

$\mathbf{q}$ àḍa $\boldsymbol{i}$ casser (son œuf) - qāḍa $\boldsymbol{u}$ défaire, séparer les parties qui tenaient ensemble

\subsubsection{Racines ambigües probablement apparentées}

Le contenu de cette sous-partie se passe de tout commentaire.

\section{- Groupe q-d-b}

qabaḍa fermer la main en contractant les doigts; resserrer le ventre, causer une constipation - qubbad sorte de tortue

qubḍān crocs enfoncés dans le murailles pour y accrocher qqch

qaḍaba et qarḍaba couper

qa'dab gros, épais; robuste, fort

qa'daba déraciner, arracher

Cette racine quadrilitère peut aussi figurer dans le groupe suivant selon l'infixe considéré. Nous l'y répétons pour qu'elle accompagne la seule autre racine qui s'y trouve.

\section{- Groupe q-d-c}

quḍā' gravier, morceaux qui se détachent du bas des murs

qaụa'a couper

qa'dab gros, épais; robuste, fort

qa'daba déraciner, arracher

18 En fait nous aurions pu écrire "la séquence qḍ” car, comme on le constatera, le corpus de cette partie ne contient aucune racine apparentée construite sur la séquence ḍq. 


\section{- Groupe q-di-m}

qadīm tout ce qui est sec et craque sous les dents

qạ̣ima grignoter, croquer du bout des dents

qarḍamä̈ couper

- Racines isolées

qaḍaf pierres minces - qaḍafä monticule sablonneux; tertre formé par les pierres et l'argile

$\checkmark$ qụf - VII. être ôté de sa place et porté ailleurs (se dit des grains de sable)

naqāyiḍ morceaux de coque d'un oeuf cassé ; fragments, morceaux cassés naqaụa disjoindre, détraquer ce qui était joint ; rompre (un contrat)

qarāyiḍ rognures, coupures

qaraḍa couper, rompre, couper en rongeant

Nous ne sommes qu'à mi-chemin et déjà cette étude devient monotone et répétitive. Les moins sceptiques de nos lecteurs sont sans doute maintenant convaincus que le lexique arabe de la pierre présente de fortes similitudes sémantiques avec son homologue latin. Mais rappelons que nous avons également la prétention d'éclaircir par cette deuxième partie des points restés obscurs dans la première. Au risque de susciter l'ennui, il nous faut donc poursuivre pour réduire par le nombre la part du hasard dans les coïncidences. La présentation de moins en moins commentée des données-sauf nécessité absolue — nous permettra d'avancer plus vite.

\section{4. raml sable}

\subsubsection{Morpho-sémantisme de la racine}

raml ${ }^{19}$ sable - ramala enrichir un tissu de perles, de pierres précieuses

'armūlä̈ chicot d'une branche qui a été séparée du tronc - 'armal veuf, veuve

Du point de vue de la forme, nous bénéficions d'un travail de recherche précédent effectué pour notre étude sur le nom arabe de la pyramide. ${ }^{20}$ Nous savons donc déjà que la racine $\sqrt{ } \mathbf{r m l}$ relève de l'étymon $\{\mathbf{r}, \mathbf{m}\}$ et que l'une des charges sémantiques de cet étymon est justement pierre.

On constate dans la racine $\sqrt{ } \mathbf{r m l}$ la présence du parallélisme sémantique pierre // couper à partir du sens de 'armūlä̈. On notera en passant que le veuvage est perçu comme une coupure du couple par l'arrachement définitif d'un de ses membres à l'autre.

On dispose de trois racines non ambigües construites sur cet étymon :

raym et raymä̈ colline, tertre; tombeau

rāma se séparer de qqn, s'éloigner (Voir annexe $\mathrm{n}^{\circ}$ A.1.S.3.)

marw silex

19 D'où l'espagnol rambla.

20 J.C. ROLLAND 2017. 
marà $\boldsymbol{i}$ donner à qqn des coups de fouet

Où l'on voit, incidemment, que les deux glides n'en font qu'un.

marmar marbre

marmara être en colère (Voir annexe, A.6.4.)

\subsubsection{Racines ambigües probablement apparentées}

\section{- Groupe r-m-}

'aram cailloux - 'iram grosse pierre destinée à indiquer le chemin dans le désert - Pl. 'urūm pierres sépulcrales des Adites

'arama mordre à qqch, enfoncer les dents dans qqch

'amarä̈ petite pierre qui indique la route

Cette racine n'a pas l'élément porter un coup > couper, mais on peut penser que le sens premier de 'amara ordonner était trancher aux sens propre et figuré. (Cf. fr. ciseau, décider).

\section{- Groupe r-m-ğ}

Les consonnes $\mathbf{r}$ et $\mathbf{m}$ étaient présentes dans les racines du groupe $\breve{\mathbf{g}}-\mathbf{r}-\mathbf{m}$ que nous avons rencontrées plus haut (2.1.2.) dans le cadre du chapitre consacré à hağar. Ces racines sont donc le résultat du croisement des étymons $\{\check{\mathbf{g}}, \mathbf{r}\}$ et $\{\mathbf{r}, \mathbf{m}\}$. Il était inutile de reproduire ici ce groupe in extenso.

\section{- Groupe r-m-h}

hūram rochers crevassés

harama couper de manière à séparer une chose d'une autre

ruhām marbre

$\sqrt{ } \mathbf{r h m}$ - II. adoucir la prononciation d'un mot en retranchant quelque son dur

\section{- Groupe r-m-d}

raḍm pierres de bâtisse (avec lesquelles on bâtit en les posant les unes sur les autres) raḍama jeter violemment à terre

ramḍā' sol jonché de cailloux, cailloux

ramāọä̈ tranchant, état de tout ce qui est tranchant, aigu

dumruz sol dur - damrazä̈ pays dont le terrain est raboteux et où l'on évite de voyager la nuit

\section{- Groupe r-m- $\boldsymbol{h}$}

hamir grande masse de sable ; gras et gros - yahmūr masse de sable hamara frapper avec violence le sol avec ses sabots (cheval) - III. enlever, emporter tout haram pyramide 


\section{- Racines isolées}

ḥimārä̈ en gén., grosse pierre; de là, grosse pierre qui ferme un réservoir d'eau et l'empêche de s'écouler au dehors, et grosse pierre qui masque la retraite du chasseur ; grosse pierre dont on ferme le tombeau

ḥamara gratter et oter la peau extérieure ; écorcher (un mouton) ; raser (la tête)

ratīmä̈ gros rocher noir

ratama briser, casser

sāmūr diamant

samara crever l'œil ; lancer une flèche

šammūr diamant

šamara lancer une flèche

șarīmä̈ monticule de sable

șarama couper, retrancher en coupant

kurtum ou kurtūm rocher, grosse pierre

kirtīm hâche, cognée pour abattre et couper du bois

murād monticule de sable

marada couper, retrancher en coupant

\subsubsection{Racines ambigües avec le seul sémantisme pierre}

\section{- Groupe r-m-}

$\sqrt{ } \mathbf{r m}^{\mathbf{c}}$ - yarma ${ }^{c}$ pierres plates, molles et friables

șam'ar sol dur et raboteux

irmis pierre

'amrät petit bijou que l'on met entre des perles enfilées pour les séparer

\section{- Racine isolée}

mart ou mirt désert, nu et humide (lieu)

\section{5. șahr roc, rocher}

\subsubsection{Morpho-sémantisme de la racine}

șahr roc, rocher, pierre énorme et très dure

Cette racine, qui n'offre pas d'autre sémantisme que celui de la roche, relève très probablement de l'étymon $\{\mathbf{h}, \mathbf{s}\} .{ }^{21}$ Cette hypothèse se vérifie par le sémantisme des quatre racines ci-dessous dont trois sont non ambigües :

21 Au sujet de cet étymon, on lira dans BOHAS \& BACHMAR 2013: 146: " Des réalisations sont attestées dans les deux sens [c'est-à-dire : h̆ h-ṣ ou ș-h h] mais il est impossible d'établir une relation entre elles. » Cette partie de notre étude apporte la preuve que cela est au contraire bel et bien possible. 
șaḩha frapper un corps dur, cogner

șayhāa dur (rocher) - șayhadūn dureté d'un rocher

Ce quadrilitère est naturellement ambigu mais il semble bien n'être qu'une extension par

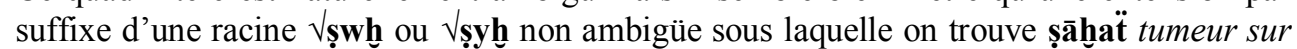
l'os causée par un coup ou une morsure

\section{husyat testicule}

hașà i couper, châtrer (un cheval entier) - hașiyy châtré, coupé ; castrat, eunuque

L'examen de ces trois racines nous permet non seulement de déterminer l'étymon de șahr avec un fort pourcentage de probablité, mais il confirme aussi la présence du parallélisme sémantique pierre // porter un coup dans une nouvelle famille, celle de l'étymon $\{\mathbf{h}, \mathbf{s}\}$. Ce n'est pas une famille très nombreuse mais on y trouve tout de même quelques racines ambigües probablement apparentées. Le parallélisme y est si explicite que nous pourrons nous passer de commentaires.

\subsubsection{Racines ambigües probablement apparentées}

\section{- Groupe ș-h̆-r}

Aux côtés de notre mot vedette, on trouve dans ce groupe, avec radicales permutées :

hirṣ digue ; chameau grand et robuste - harīṣ bord d'un fleuve ; île

hirṣ lance mince - harṣ lance, fer de lance - harīs fer de lance

harbașị pierre très petite et luisante qu'on rencontre dans le sable ; coquillage (conque de Vénus)

harbașa séparer plusieurs choses les unes des autres

À noter tout particulièrement :

- La présence d'un nouveau coquillage.

- Une nouvelle apparition du bord ou rivage.

\section{- Groupe ș-h-m}

șambāät sol pierreux

șamaha blesser qqn à la cavité de l'oreille ; donner un coup de poing sur qqch de creux șahmā' terrain pierreux

hasạama - VIII. couper (se dit d'un sabre qui coupe son fourreau)

\section{- Racine isolée}

šahuṣa être une masse - šaxīṣ dur, désagréable (parole)

لšhṣ - IV. effrayer, épouvanter qqn (Cf. annexe, A.1.S.4.) ; manquer le but en lançant une flèche (Cf. annexe, A.2.6.) 


\section{6. ğundul ou ğandal pierre, rocher}

\subsubsection{Morpho-sémantisme de la racine}

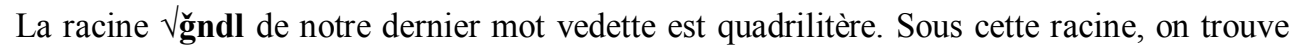
également le verbe ğandala jeter par terre. Du point de vue morphologique, elle peut être considérée comme une extension par infixe nasal de la racine $\sqrt{\mathbf{g} d l}$ sous laquelle on trouve le verbe ğadala qui signifie lui aussi jeter par terre. Et jeter quelque chose, c'est s'en séparer, comme nous l'avons déjà vu à plusieurs reprises dans les pages précédentes. On constate donc dans la racine $\sqrt{\mathbf{g} n d l}$ la présence du parallélisme sémantique pierre // porter un coup. Reste à déterminer l'étymon de cette racine.

La première combinaison théorique, $\{\check{\mathbf{g}}, \mathbf{d}\}$, est assez fructueuse pour que nous puissions nous passer d'examiner quels résultats donneraient les deux autres, d'autant plus en sachant que dans la théorie de Bohas, cet étymon, doté de la charge sémantique porter un coup, relève de la matrice 5 \{[coronal],[dorsal]\} porter un ou des coups.

Construites sur cet étymon, on dispose effectivement de deux racines non ambigües :

- La racine sourde $\sqrt{\mathbf{g}} \mathbf{d d}$

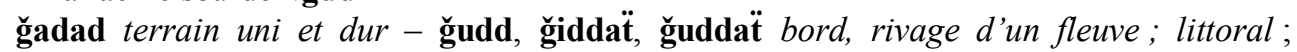
Djedda

Notons cette troisième apparition du bord ou rivage (cf. 2.1.2. groupe ğ-r-f, p. 385; 2.5.2. groupe ș-hb-r, p. 394).

ğadda couper, retrancher, tailler

- La racine quadrilitère à redoublement $\sqrt{\breve{g} d g ̆ d ~}$

ğadğad, ğadğadä̈ sol plat et dur

ğudğud inflammation de l'œil, pustule qui naît à la naissance de la prunelle

Troisième apparition de la pustule, symptôme d'une maladie de la peau (cf. 2.1.2. groupe ğ-r-d, p. 384 et groupe ğ-r-m, p. 386)

Dans les deux racines, le parallélisme sémantique pierre // porter un coup est patent.

\subsubsection{Racines ambigües probablement apparentées}

La plupart des items se passent de commentaires.

\section{- Groupe ğ-d-I}

Aux côtés de notre racine vedette, on trouve d'abord dans ce groupe la racine $\sqrt{\mathbf{g}} \mathbf{d l}$ dont on a vu qu'elle en est probablement à l'origine :

ğadl dur ; tombeau - ğadil dur ; fort, robuste - ğadālä̈ terre couverte d'un sable fin ğadala jeter, renverser par terre

Cette racine fertile est aussi probablement à l'origine d'une autre racine quadrilitère par insertion d'un infixe guttural :

ğaḥdal ou ğuhdul grand et gros; fort, robuste

ğạ̣dala renverser, jeter par terre

Avec radicales permutées :

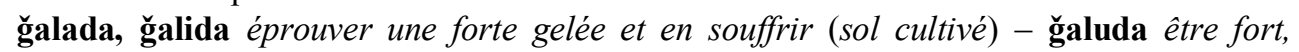
robuste, dur- ğalad cuir, peau; dureté, endurcissement ; terrain uni et dur- galìd gelée 
araméen gilda testicule

ğalada frapper sur la peau et l'endommager, fouetter qqn ; écorcher une pièce de bétail ; renverser, jeter qqn à terre

Quant au nom quadrilitère ğalmad ou ğalmūd grosse pierre, rocher, apparemment sans correspondant verbal, il peut être associé aussi bien à ce groupe $(\sqrt{g} \mathbf{g} l \mathbf{d}+$ infixe $\mathbf{m})$ qu'au suivant $(\sqrt{\mathbf{g}} \mathbf{m d}+$ infixe $\mathbf{l})$.

\section{- Groupe ğ-d-m}

ğamd gelée, glace, solide (non liquide) - ̌̆umd sol élevé et dur - ğamad glace, sol élevé et dur

ğamada couper

ğalmad, ğalmūd grosse pierre, rocher

dimā

\section{- Groupe ğ-d-r}

ğadara avoir des pustules, s'en couvrir ; contracter des callosités

ğadara faire élever une muraille autour de qqch - ğadr mur, muraille - ğadīrä̈ enclos fait de pierres pour les bestiaux

ğurdä̈ sol uni et nu, où rien ne crô̂t

ğarada oter, enlever (p. ex. les feuilles des arbres, la peau, etc.)

Nous avons déjà rencontré ce groupe en 2.1.2. On peut en déduire que ces deux racines résultent du croisement des étymons synonymes $\{\check{\mathbf{g}}, \mathbf{r}\}$ et $\{\check{\mathbf{g}}, \mathbf{d}\}$ porter un coup.

- Racines isolées :

ğadaf tombeau

ğadafa couper un membre du corps

ğahād sol dur et stérile

ğihād lutte, combat (Cf. annexe, A.6.1.)

ğadb qui souffre de la stérilité (lieu, pays, sol)

akkadien (a)gadibb- $s o c$ - éthiopien gadb hache

\subsubsection{Racines ambigües avec le seul sémantisme pierre}

\section{- Groupe ğ-d-s}

ğādis dur et ferme; terrain sec, dur, inculte

'asğad perles ; chameau gros, épais

\section{- Groupe ğ-d-n}

ğanad terrain inégal et rocailleux

danağa fixer, raffermir solidement 
- Racines isolées :

'ağad solide

ğadaš sol dur

Nous voici enfin arrivés au terme de cette partie de notre étude consacrée aux dénominations arabes de la pierre. Avant de faire la synthèse de nos observations, il nous a paru utile d'ajouter quelques racines supplémentaires dans lesquelles on observe le même parallélisme sémantique que nous avons relevé dans nos six mots vedettes et leurs apparentés :

barada limer

barad grêle

Les deux mots sont toujours usuels.

balāt pavé

Mot usuel. L'arabe classique a connu balața frapper qqn à l'oreille avec le bout de l'index - III. se battre au sabre ou au bâton

șawt 'ağašš voix rocailleuse

Mot usuel. L'arabe classique a connu ğašš endroit pierreux, inégal et dur - ğaššā' sol semé de caillous // ğašša briser, casser, broyer

a'bal rocher blanc, granit

Mot usuel. L'arabe classique a connu 'abala couper, retrancher

'alaba couper, retrancher, abattre

‘ulb sol dur et stérile

Les deux mots sont obsolètes.

faḍda casser, rompre, briser

Mot usuel. L'arabe classique a connu faḍḍä terrain rocailleux

mahw petites pierres minces, brillantes et transparentes ; perles

mahā $\boldsymbol{u}$ porter à qqn un coup violent

Les deux mots sont obsolètes.

\subsection{Synthèse de nos observations}

Dès notre introduction, sans être géologue ni minéralogiste et sans pousser jusqu'à la lune, ${ }^{22}$ la simple connaissance du monde nous avait permis d'énumérer, du grain de sable au rocher, divers types de pierre en fonction de leurs volumes, de leurs formes ou de leurs usages, depuis la meule du meunier jusqu'à l'édification des digues, routes, ponts, murs, tombes et pyramides. Il semble qu'avec les six mots arabes que nous avons mis en vedettes et leurs apparentés respectifs nous en ayons fait un honnête inventaire. Ces six mots du langage usuel nous ont même amené à rencontrer en route des termes plus spécifiques comme le charbon, le marbre, le silex et le granit.

22 Nous aurions pu : dans l'Univers, la lune n'est qu'un gros grain de sable, une "poussière d'étoile"; on peut considérer le nom qa mar comme construit sur l'étymon $\{\mathbf{r}, \mathbf{m}\}$. (Voir 2.4.1.). Voir aussi en 3.2. la racine BDR. 
Nous avions également énuméré divers objets pouvant être assimilés ou comparés à des pierres : noyaux, dents, fragment d'os, coraux, coquilles d'œuf, coquillages, testicules... Ils ont tous apparu, plus quelques autres auxquels nous n'avions pas d'abord pensé :

- en premier lieu le sol dur, sec, stérile, où rien ne pousse ; ce n'est pas de la pierre mais c'est tout comme; le désert, en somme ;

- les surfaces gelées, la glace, le verglas ;

- les perles, la carapace de la tortue ;

- les callosités de la peau, le poing fermé et même le ventre constipé.

Nous savions que la pierre était symbole de dureté, plus encore en arabe-elle est omniprésente dans le monde arabophone - qu'en indo-européen où l'arbre en général et le chêne en particulier lui disputent ce rôle. ${ }^{23}$ Nous avons découvert que cette caractéristique naturelle se déploie en un faisceau de dérivations sémantiques d'ordre physique ou moral : solidité, force, robustesse, grosseur, grandeur, épaisseur, hauteur ... Les hommes et les chameaux qui "en imposent" sont définis par des mots évocateurs d'un nom de la pierre ; on dit bien en français "Cet homme, c'est un roc."24

Quant au rapport sémantique de la pierre avec l'action de porter un coup, qui parcourt toute cette étude, il faut bien reconnaître qu'il n'est pas très surprenant: nous avions d'emblée rappelé que la pierre taille et surtout qu'elle est taillée. D'où, semble-t-il, le peu de cas fait de ce rapport par l'étymologie traditionnelle dans la paire rūpēs // rumpō, sans doute considéré comme une évidence ne méritant pas une attention particulière.

Ce rapport n'allait pas forcément de soi pour l'arabe : sauf à avoir lu quelque part et il y a bien longtemps que si le nom arabe de l'île est dérivé d'une racine ayant le sens de couper, c'est parce qu'une île est ou semble coupée du continent. Mais une île n'est pas une pierre, ce n'est qu'une métaphore de la pierre. Le nombre de fois où nous avons pu vérifier ici la réalité du rapport pierre // porter un coup en arabe confirme son existence à la fois en sémitique - où le cas de l'île est loin d'être le plus flagrant—et en indo-européen pour la paire gréco-latine lapis // $\lambda \dot{\varepsilon} \pi \omega$. Nous savons maintenant que, depuis les origines du langage, la pierre a dû être considérée comme le résultat d'une fragmentation : les hommes ont très vite compris que le destin du rocher était un tas de sable.

Mais l'intérêt de notre étude pour l'étymologie indo-européenne ne se limitera pas à cette confirmation. Nous avons en effet la hardiesse de penser que nos observations pourraient aussi apporter quelque lumière sur les origines des mots grecs $\lambda \dot{\lambda} \boldsymbol{\theta} \boldsymbol{\sigma} \varsigma$ et $\boldsymbol{\pi} \dot{\varepsilon} \tau \boldsymbol{\rho} \overline{\boldsymbol{\alpha}}$ sur lesquels, un peu moins démuni qu'au début de notre recherche, nous allons maintenant revenir.

23 Le français $d u r$ et l'anglais tree sont issus de la racine IE *deru- « dur ».

24 «Le docteur [...] se dresse, calme et puissant, au-dessus de cette petite épave; c'est un roc », J.-P. SARTRE, La Nausée, Paris: Gallimard, 1938: 94. 


\section{Retour sur $\lambda i ́ \theta 0 \varsigma$ [líthos] et тв́трā [pétrā]}

\section{1. grec $\lambda i ́ \theta o s$ [líthos]}

Puisque le grec $\boldsymbol{\lambda} \mathbf{i} \boldsymbol{\theta} \mathbf{o}$ s est sans famille dans sa propre langue, le cas du couple lapis // $\boldsymbol{\lambda} \boldsymbol{\varepsilon} \boldsymbol{\pi} \boldsymbol{\omega}$ nous incite à aller voir du côté du latin. On y tombe d'emblée sur un mot qui s'en rapproche fort, du moins dans la forme : lìtus, -oris rivage de la mer, côte, littoral. Et c'est sans grande surprise qu'on lit à son propos dans le DELL : « Aucun rappochement sûr ».

Et pourtant, rappelons ces paires que nous avons trouvées pour l'arabe :

ğurf, ğuruf berge, bord rongé par l'eau ğarafa enlever, emporter tout, en balayant, d'un coup de balai ou de pelle

harīṣ bord d'un fleuve harị̣̄ fer de lance

ğudd, ğiddä̈, ğuddä̈ bord, rivage d'un fleuve; littoral ğadda couper, retrancher, tailler

șawh berge, bord d'une rivière, bord élevé comme un mur șāḥa fendre

À quoi nous pouvons ajouter le tigré gərgər rocher, falaise et au moins une racine arabe associant clairement la pierre au bord:

șibr ou șubr marge, bord // șubr ou șubur ou șabbārä̈ terrain couvert de petits caillous - șabar glace, eau gelée - șibārä̈ ou șabārä̈ pierres ; éclat, morceau de rocher ou de fer-șubrä̈ pierres dures - șabīr colline rocailleuse

À l'évidence, l'arabe perçoit la rive comme une coupure. Tel le vent frappant les pics des sommets, l'eau des oueds et des océans, repue d'une terre friable promptement engloutie et dissoute, se heurte à la roche des rives ou des rivages, la longe, la ronge et lentement la façonne. Le lexique arabe apporte la preuve qu'il y a bel et bien un lien sémantique fort entre les mots qui désignent la pierre et les signifiants de la rive, entre les noms de la dune ou de la digue et ceux du littoral. Et donc, très probablement, en indo-européen aussi, entre le grec $\boldsymbol{\lambda} \mathbf{i} \boldsymbol{\theta} \mathbf{s}$ et le latin lïtus.

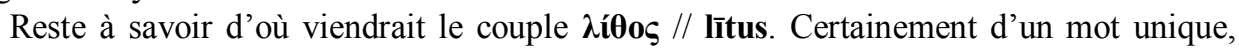
seul ou rare vestige d'une langue méditerranéenne depuis longtemps disparue, à moins qu'il ne soit issu de la branche celtique d'une racine indo-européenne en *pl- comme *plēdéchirer ou *pel- peau, peler dont le thème *pel-(i)-s- rocher-au vu de ce que nous savons maintenant-n'est probablement qu'une extension. Notons avec prudence que l'hypothèse celtique pourrait convenir pour les mots germaniques désignant le plomb, tels l'anglais lead, également d'origine incertaine. En glosant $\boldsymbol{\pi} \varepsilon \dot{\lambda} \lambda \boldsymbol{\alpha}$ [pélla] par $\lambda \mathbf{i} \boldsymbol{\theta} \boldsymbol{\alpha}$, Hésychios nous donnait peut-être sans s'en douter la clef de leur commune origine.

Notons que l'ordre *pl- de ces racines est exactement l'inverse de celui que nous avons retenu plus haut comme étant à la source de lapis et des ses apparentés grecs et latins. Comme nous l'avons constaté à plusieurs reprises en d'autres occasions, on voit une fois de plus que le non ordonnancement des consonnes radicales n'est pas propre au domaine sémitique. 


\section{2. grec пв́тра̄ [pétrā]}

Le cas de $\boldsymbol{\pi} \dot{\boldsymbol{\varepsilon}} \boldsymbol{\tau} \boldsymbol{\rho} \overline{\boldsymbol{\alpha}}$ est plus problématique. Ce mot est non seulement sans famille grecque mais on ne voit pas quel mot latin pourrait lui être apparenté. ${ }^{25}$ Watkins le pense dérivé de la racine *per- 2 conduire, passer par-dessus via une forme suffixée *per-trā- mais le lien sémantique proposé par cet auteur est forcé et peu convaincant. ${ }^{26}$

Dans ces cas-là, notre réaction habituelle est de nous tourner vers le sémitique, ne serait-ce que pour vérifier s'il n'y aurait pas quelque possibilité de rapprochement possible, qu'il soit savant ou naï. Notons que nous avons en cette matière et sur le même thème d'éminents modèles :

- Chantraine opte pour une origine sémitique du grec $\beta \mathbf{\beta} \mathbf{l} \boldsymbol{\alpha} \alpha \mathbf{\omega} \boldsymbol{\omega} \boldsymbol{0}$ [bizakíōn] petits cailloux dont l'étymon pourrait être l'araméen $\mathbf{b i z q} \overline{\mathbf{a}}^{27}$ débris, petite pierre ;

- Michel Masson ${ }^{28}$ propose de rapprocher le grec $\chi \boldsymbol{\alpha} \lambda \boldsymbol{\lambda} \mathbf{\xi}$ [kháliks] caillou, gravier et ses

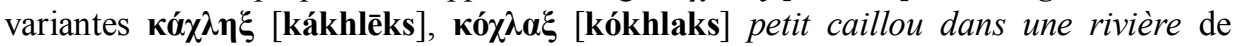
l'hébreu halluq caillou dans une rivière.

Nous voilà doublement encouragé à proposer une origine sémitique du mot grec $\pi \dot{\varepsilon} \tau \boldsymbol{\rho} \overline{\boldsymbol{\alpha}}$. Nous allons dire sur quoi nous fondons cette hypothèse.

1. Phonétiquement, nous savons par d'autres cas d'emprunts similaires,

- qu'un $\boldsymbol{\pi}$ - initial correspond généralement à un $\mathbf{p}$ - en akkadien et à un $\mathbf{f}$ - en arabe ;

- qu'en deuxième position, une dentale ou une interdentale peut alterner avec une sifflante ;

- que cette deuxième radicale peut être sourde, sonore ou emphatique.

2. Sémantiquement, nous savons maintenant qu'un nom sémitique de la pierre a de fortes chances de relever d'une racine dont le sens premier est porter un coup.

À la fin de la sous-partie 2.6., nous avions relevé une paire correspondant aux critères que nous venons de poser :

faḍdät terrain rocailleux, élevé // faḍda casser, rompre, briser

En poursuivant notre cueillette, nous en avons glané d'autres :

fatta écraser, broyer qqch entre ses doigts ; fendre (les pierres)

fadara - II. se casser, être brisé, cassé en petits et grands morceaux - fādirä̈ rocher détaché au haut d'une montagne

fadfad sol uni et dur // fadfada courir en se sauvant devant l'ennemi

fusayfisā' coquillage ; mosaïque // fasfas sabre émoussé (sabre)

Vf̣̣l - mafșil monceau oblong de sable ; tas de silex // fașala séparer

25 À moins d'être très audacieux et de reconnaître dans $\pi \dot{\varepsilon} \tau \rho-$ mais dans un ordre différent les mêmes consonnes que celles du radical latin rupt- de certaines formes dérivées du verbe rumpo...

26 "With possible earlier meaning bed-rock (< what one comes through to)".

27 Notons au passage que ce mot présente toutes les qualités requises pour être intégré à ce que nous appellerons plus loin la famille élargie de $\pi \dot{\varepsilon} \tau \boldsymbol{\tilde { \alpha }} \mathbf{~}:$ il a une labiale à l'initiale suivie d'une sifflante et est dérivé d'un verbe $\mathbf{b}^{\mathbf{e}} \mathbf{z a q}$ qui a le sens de briser, broyer.

28 MASSON 2013a: 217-218

j고. 17 (2017): 377-406 
fașan/fașà pépin de raisin sec // fașà détacher, séparer

fașị̦ noyau // fașșa séparer, disjoindre

fașịt pelures de dattes, rognures d'ongles

Voilà donc un ensemble non négligeable de neuf racines - dont six non ambigües - où il est question à la fois de pierres (ou d'objets assimilés à des pierres) et de coupures. Ces racines ne sont pas construites sur le même étymon mais leurs étymons relèvent tous de la matrice phonique $\mathrm{n}^{\mathrm{o}} 1$ \{[labial],[coronal]\} porter un coup ou des coups.

En poussant la recherche, on trouve encore cinq racines non ambigües mais orphelines de l'élément pierre:

fatta - VII. être cassé, brisé

fadda être tout seul, isolé, séparé des autres

fazza faire défection et se séparer de qqn

fazfaza donner la chasse à quelqu'un et l'éloigner

fațā donner une chasse vigoureuse à un animal

... et une trentaine de racines ambigües, elles aussi orphelines de l'élément pierre:

fata'a casser

fataha ouvrir - IV. trancher (dans une dispute)

fatrașa couper

fatag்a fouler avec les pieds au point d'écraser

fataqa fendre, rompre; séparer, défaire, découdre

fataġa casser, briser

fadaha casser, briser, écraser avec une pierre

fadaša casser, briser, écraser

fadaġa casser, briser, écraser

faduma être raboteux, avoir la surface couverte d'aspérités

fadaha - V. écarter les jambes

fazara rosser, donner des coups de bâton sur le dos; déchirer un habit

faza'a - II et IV. effrayer

fasa'a déchirer, lacérer ; donner à quel- qu'un des coups de bâton sur le dos

fasağa - II. écarter les cuisses

fasaḥa élargir

fasaha disjoindre, séparer

fasaqa sortir de son enveloppe (datte mûre)

fașaha démettre un membre du corps

fașada ouvrir une veine

fașa'a presser une datte verte pour la faire sortir de son enveloppe

fașama casser, déchirer qqch

fadaha casser (un morceau de bois ); crever un oil

faḍaġa casser (un morceau de bois)

fața'a frapper qqn sur le dos

fațaha frapper qqn avec un bâton

fațada éloigner qqn

fatara fendre, pourfendre, couper en deux

fațama sevrer un enfant; couper en faisant une incision

Ces racines, on le voit, ne nous offrent pas de dérivé "pierreux" mais il serait bien étonnant, au moins pour certaines d'entre elles, qu'un tel vocable n'apparaisse pas dans une forme dialectale ou dans une autre langue sémitique. Notons déjà les racines fadara, fațara et fatrașa; dans notre recherche d'éventuels cognats sémitiques du grec $\pi \dot{\varepsilon} \tau \rho \overline{\boldsymbol{\alpha}}$, ce sont de bons candidats. 
Venons-en à l'akkadien. Nos glanures y sont moins nombreuses mais on trouve tout de même :

pasāsu effacer, oblitérer ; détruire, aplatir, raser

pașādu trancher, couper, tailler, entailler

Page | 402

patāhu percer (mur, four, partie du corps), crever, piquer, ouvrir, forer, sonder ; (bétail)

poignarder, enfoncer un couteau, frapper (et tuer) avec un couteau

patarru massue, masse d'arme en cuivre ou en bronze

patru épée, dague ; couteau de boucher, de tanneur, ...

pattu frontière

patû défaire ; (orge) enlever la balle, décortiquer, enlever les écales ; percer

pațāru détacher, défaire; quitter (un lieu); découvrir (une partie du corps); disperser

(des troupes) ; rompre (un contrat) ; dissiper ; annuler, interrompre ; dételer ; séparer,

découper ; écarter, enlever, disperser ; démonter, desserrer, défaire pāṭu frontière, limite

Nous n'avons pas de commentaires à faire ici autres que ceux que nous avons déjà faits pour l'arabe. Comme bons candidats à une éventuelle et plus proche parenté avec $\boldsymbol{\pi} \dot{\varepsilon} \tau \boldsymbol{\rho} \overline{\boldsymbol{\alpha}}$, notons les noms patarru et patru, et le verbe pațāru, qui est d'ailleurs de la même racine sémitique que l'arabe fațara.

Pour tenter de convaincre les plus sceptiques, nous pourrions aligner des listes similaires avec la labiale b- à l'initiale au lieu du f-. En arabe comme en akkadien, pour certaines des racines ci-dessus, il existe en effet des variantes en $\mathbf{b}-\mathbf{C}^{2}-\mathbf{C}^{3}$. Nous nous contenterons, pour donner un peu plus de corps à ce que nous pensons être la famille nucléaire de $\pi \hat{\varepsilon} \tau \rho \overline{\boldsymbol{\alpha}}$, de signaler quelques racines sémitiques en $\mathbf{b}-\mathbf{C}^{2}-\mathbf{r}$ où $\mathbf{C}^{2}$ est une dentale ou une interdentale. Notre référence sera le fascicule 2 du Dictionnaire des racines sémitiques :

BDR (p. 46)

"Pour Dillmann, le sens premier est couper". On y retrouve incidemment la lune, mais ici sous la forme badr. Cf. qamar, 2.7., note de bas de page.

BĐR (p. 47)

badara disperser, disséminer

BṬR (p. 61)

bațara fendre, ouvrir une plaie, percer un ulcère, etc.

al-Bațā' (nom de la ville de) Pétra

On est en droit de se demander si ce toponyme est bien un emprunt au grec, comme le veut la tradition, ou un simple dérivé de $\sqrt{ } \mathbf{b t ̣ r}$.

bayțân $\mathbf{r}^{27}$ médecin-vétérinaire

27 Pour certains, ce mot serait issu du grec i i $\pi \iota \alpha \tau \rho o ́ \varsigma$ [hippiatrós], littéralement «médecin spécialiste des chevaux ». Pour d'autres, du latin veterinarius, «relatif aux bêtes de somme, vétérinaire ; médecinvétérinaire ». Mais le rapport sémantique entre veterinarius et vetus, « vieux » n'est pas évident. Columelle, célèbre agronome romain du $1^{\text {er }}$ siècle, utilise deux fois le mot veterinarius dans son œuvre. Après quelques années passées dans l'armée, où il occupe le poste de tribun en Syrie en 35, il se con- 
Probable extension de $\sqrt{ } \mathbf{b t ̣}$ par infixation du glide.

BDR (p. 78)

La dentale de cette racine se réalise en sifflante emphatique dans plusieurs langues sémitiques, d'où

bașara couper, retrancher // bușr pierres dures et blanches; marge, bord; écorce bașrä pierres dures et blanches; terrain dur, pierreux et dont on tire les pierres - alBașrät Basra ou Bassorah.

Dans les textes anciens, les noms de Pétra et Basra sont souvent associés, comme s'ils avaient plus ou moins le même sens.

akkadien bașāru couper, déchirer

hébreu bișșēr couper

En sud-arabique, on trouve diverses formes avec dentale et le sens de couper, déchirer.

NB : Il n'aura pas échappé aux plus attentifs de nos lecteurs qu'une racine rencontrée plus haut présente, au prix d'une métathèse, de forts signes de parenté avec celle-ci. Rappelons-la :

șibr ou șubr marge, bord // șubr ou șubur ou șabbārä̈ terrain couvert de petits caillous șabar glace, eau gelée - șibārä̈ ou șabārä̈ pierres ; éclat, morceau de rocher ou de fer șubrat pierres dures - șabīr colline rocailleuse

En veut-on une autre, proche de cette dernière?

zabara éloigner, repousser qqn de qqch // zabr pierres dures - zubrä̈ morceau, fragment; morceau de fer

BTR (p. 90)

batara couper la queue d'un animal, l'écourter

akkadien butturu mutiler

amharique bättor bâton

araméen $\mathbf{b}^{\mathbf{e}}$ tar, bitrā morceau

BFR (p. 91)

batara être couvert de pustules, de boutons // bațr terrain sablonneux parsemé de pierres blanches

Nous espérons, par ces quelques pages, avoir apporté notre pierre à la recherche étymologique ; l'avenir dira si ce travail s'apparente plus au sable qu'au rocher.

sacre à l'agriculture. Cela ne suffit sans doute pas pour prouver que le mot est un emprunt à une langue parlée aux frontières de la Syrie à l'époque de Columelle mais on n'a pas d'autres attestations. Nous penchons donc plutôt pour un emprunt par le latin à une langue sémitique d'un cognat de bayțār. 


\section{Bibliographie}

Association Assyrophile De France. 2017. Dictionnaire akkadien. En ligne sur $<\mathrm{http} / / \mathrm{www}$. assyrianlanguages.org/akkadian/index_en.php>.

BAILly, Anatole. 1901. Dictionnaire grec-français. Hachette, Paris.

BELot, Jean-Baptiste. 1955. Dictionnaire arabe-français « El-faraïd». Imprimerie catholique, Beyrouth.

BoHAS, Georges. 1997. Matrices, étymons, racines. Peeters, Leuven-Paris.

— / BACHMAR, Karim. 2013. Les étymons en arabe: Analyse formelle et sémantique. (Recherches, $\mathrm{n}^{\circ}$ 23). Dar El-Machreq, Beyrouth.

- / SAguer, Abderrahim. 2012. Le son et le sens: Fragment d'un dictionnaire étymologique de l'arabe classique. Presses de l'IFPO, Damas.

- / SAguer, Abderrahim. 2014. The Explanation of Homonymy in the Lexicon of Arabic. ENS Éditions, Lyon.

CAILlois, Roger. 1966. Pierres. Gallimard, Paris.

Chantraine, Pierre. 1977. Dictionnaire étymologique de la langue grecque. Klincksieck, Paris.

CoHEN, David. 1970- . Dictionnaire des racines sémitiques ou attestées dans les langues sémitiques. Fasc. 1 et 2: Paris / La Haye: Mouton, 1970 ; fasc. 3 à 10 (avec la collaboration de F. Bron et A. LONNET): Louvain / Paris: Peeters, 1993-2012.

De VAAn, Michiel. 2008. Etymological Dictionary of Latin and the Other Italic Languages. Brill, Leyde.

Dictionnaire akkadien $\rightarrow$ AsSOCIATION ASSYROPHILE DE FRANCE.

ERnOUt, Alfred / MEILLET, Antoine. 1932, réédition 2000. Dictionnaire étymologique de la langue latine. Klincksieck, Paris.

ETYMONLINE : abréviation usuelle de Online Etymology Dictionary (en ligne).

FOURNET, Arnaud. 2008. «La reconstruction de l'indo-européen et la réalité du sémitique : convergences et perspectives ». Langues et Littératures du Monde Arabe, 7: 3-39.

GAFFIOT, Félix. 1934. Dictionnaire latin-français. Hachette, Paris.

Guiraud, Pierre. 1982, 1994. Dictionnaire des étymologies obscures. Payot, Paris.

IBN MANZ̄ŪR (XIIIe s.), Lisān al- 'Arab. (en ligne sur http://www.baheth.info/index.jsp).

Indo-European Lexicon, Pokorny Master PIE Etyma, The College of Liberal Arts, University of Texas, Austin.

KaZimirski, A. de Biberstein. 1860. Dictionnaire arabe-français. Maisonneuve et Cie, Paris.

KHATEF, Laïla. 2004. «Le croisement des étymons : organisation formelle et sémantique ». Langues et Littératures du Monde Arabe, 4: 119-138.

Le Trésor de la Langue Française. http://www.cnrtl.fr/

Masson, Michel. 2013a. Du sémitique en grec. Éditions alfAbarre, Paris.

— . 2013b. «Perles, coraux et bilitères ». Semitica et Classica, 6: 269-278.

REIG, Daniel. 1983. Dictionnaire arabe-français / français-arabe «As-Sabil ». Librairie Larousse, Paris.

Rolland, Jean-Claude. 2015. Étymologie arabe: dictionnaire des mots de l'arabe moderne d'origine non sémitique. L'Asiathèque, Paris.

jais 17 (2017): 377-406 
— . 2017. Dix études de lexicologie arabe. $2^{\mathrm{e}}$ édition. Meaux, J.C. Rolland.

WATKIns, Calvert. 2000. The American Heritage Dictionary of Indo-European Roots. Second Edition. Houghton Mifflin Company, Boston \& New York.

WeHr, Hans. 1966. A Dictionary of Modern Written Arabic / edited by J. Milton Cowan. Cornell University Press, Ithaca NY.

(c) Jean-Claude Rolland, Meaux / France 4 jclrolland@hotmail.com 


\title{
Annexe : Le réseau sémantique de " porter un coup »
}

\author{
(extrait de BoHAS \& SAgUeR 2012: 220 sq.)
}

A. Porter un coup ou des coups (sans spécifier l'objet)

A.1 Frapper avec un objet tranchant, de là :

A.1.1 l'objet ou une partie de l'objet (sabre, lame, hache, etc.)

A.1.2 spécification: fendre, déchirer, inciser, mordre, ouvrir, etc.

A.1.3 résultat de l'action : la partie par rapport au tout :

A.1.3.1 raccourcir, tronquer

A.1.3.2 tuer, massacrer > mourir, achever, terminer, fin, bout...

A.1.3.3 raser, peler, racler, écorcher, dépouiller, enlever, arracher

A.1.3.4 couper, séparer une partie du tout, emmener une partie

A.1.3.4.1 petite quantité, portion, tranche

A.1.3.4.2 être mis à l'écart, isolé, seul. Cette orientation donne lieu à une masse de sens qui tournent tous autour de l'idée "séparer, se séparer, (se) disperser" que nous appellerons A.1.S., qui se ramifie de la manière suivante :

A.1.S.1 (se) disperser, (se) répandre, semer
$>$ divulguer un secret

$>$ dilapider ses biens

A.1.S.2 éloigner, repousser, détourner

A.1.S.3 réfléchi: se séparer, s'éloigner

A.1.S.3.1 modalité de la séparation : marcher, fuir, courir > rapidité

A.1.S.4 causativité : faire partir, chasser, effrayer

A.2 Frapper avec un objet pointu
A.2.1 l'objet ou une partie de l'objet (lance, flèche, pointe, etc.)
A.2.2 donner un coup de lance, percer, pénétrer, ...
A.2.3 sortir de, émerger, pousser, être saillant, être au sommet
A.2.4 sonder
A.2.5 ficher, planter dans la terre
A.2.6 se planter dans l'objectif, atteindre ou manquer le but; de là : avoir tort ou raison

A.3 Frapper avec un fouet, un bâton, un objet quelconque

A.3.1 l'objet

A.4 Blessures diverses consécutives à des coups

A.5 Préparation de l'action : aiguiser, affiler...

A.6 Réciprocité
A.6.1 se battre, attaquer
A.6.3 victoire ou défaite
A.6.2 faire la guerre
A.6.4 s'irriter, être violent

A.7 Frapper avec la main, le pied ou diverses parties du corps
A.7.1 pousser, repousser
A.7.2 protéger, conserver, garder

B. Conséquence immédiate de $A$
B.1 Briser, casser, piler
B.2 Détruire, périr, faire périr, perdre

jais 17 (2017): 377-406 